Classification

Physics Abstracts

$07.85-47.55 \mathrm{M}-81.70 \mathrm{C}$

\title{
Measurement of Diffusion in Liquids within Porous Solids by X-Ray Microradiography: Determination of Errors and Optimization of Experimental Conditions
}

\author{
Stephanie E.P. Dowker $\left({ }^{1}\right)$, Paul Anderson $\left({ }^{2}\right)$ and James C. Elliott $\left({ }^{2}\right)$ \\ ( ${ }^{1}$ Department of Conservative Dentistry \\ $\left({ }^{2}\right)$ Department of Child Dental Health, The London Hospital Medical College, Turner Street, \\ London E1 2AD, UK
}

(Received January 2; accepted January 20, 1995)

\begin{abstract}
In principle, X-ray absorption measurements provide a straightforward method for determining local concentrations and fluxes, and hence local diffusion coefficients, during diffusion of species with high atomic number, within liquids, gels or opaque porous solids. However the application of this method is limited by the errors arising from X-ray photon statistics, which cannot be calculated exactly by analytical methods. Numerical simulation has been used to investigate the influence of experimental variables on the errors in diffusion coefficients determined by an application of Fick's 1st Law. From these, guidelines have been developed for optimization of experimental conditions.
\end{abstract}

\section{Introduction}

Direct measurements of the evolution of the concentration:distance profile during diffusion in response to a change in the external concentration have been made within liquids by optical absorption spectrometry $[1,2]$ and within gels with a synchrotron X-ray fluorescence microprobe [3]. Direct measurements from concentration profiles have also been made for porous solids using optical techniques, an X-ray absorption method, NMR, and ESR spin mapping [4]. In general, the methods for direct measurement are more complicated than methods in which the effective diffusion coefficient is determined from bulk effects, such as the change in mass of a sample or in external solution concentration during effusion [5]. However these indirect methods are only generally applicable to materials for which the effective diffusion coefficient is independent of position. The inherent advantage of direct measurement is of particular importance for materials in which characteristics of the pore network vary with position. For example, the pore size distribution within a carious lesion of dental enamel varies with distance from the natural surface of the tooth [6]. To determine local transport in such materials, direct local measurements are needed. 
The essential requirement for an X-ray absorption technique is a substantial difference in crosssections for X-ray absorption between the atoms of an element in the diffusing species (solute) and of those of the solvent and permeable medium. An example of the method is the imaging of the spread of X-ray contrast media within biporous solids [7-9]. This allowed distinction to be made between transport limited by diffusion within intracrystalline micropores and that limited by mass transport within the pores between contacting crystals. Recently, Rondot et al. [10] have used an X-ray projection microscope equipped with a charge-coupled device camera to produce a time series of microradiographic images, with a lateral resolution of $10 \mu \mathrm{m}$, of $\mathrm{Zn}^{2+}$ ions diffusing in aqueous hydrochloric acid solution during corrosion of zinc foil and pellets. Spatial distributions of concentration were quantified from the images. In another example of a quantitative microradiographic technique, a thin section of material was stepped across a fine X-ray beam and the absorption measured at each position [11]. Thus projected mass profiles of a diffusing species were sampled at known positions and after known time intervals. This method also has much improved spatial resolution (currently $\sim 10 \mu \mathrm{m}$ ) compared with the estimate of $\sim 0.5 \mathrm{~mm}$ given [4] for the classical photographic technique. However, the implementation [11] using a laboratory X-ray generator (e.g. Hilger \& Watts Y33 microfocus generator, Mo target, $35 \mathrm{kV}, 2.0 \mathrm{~mA}$ and $100 \mu \mathrm{m}$ foreshortened source), has the drawback that the usable intensity is relatively low. Typical count rates are $3 \times 10^{4}$ photons $\mathrm{s}^{-1}$ for a $30 \mu \mathrm{m}$ aperture $30 \mathrm{~mm}$ from the source with a high purity $\mathrm{Ge}$ detector and single channel analyser centred on the $\mathrm{MoK} \alpha$ line. Thus there is a substantial statistical uncertainty for each intensity measurement (standard deviation $\sim 0.2 \%$ for a $10 \mathrm{~s}$ count), unless the counting time is prolonged, for which there is limited scope if the profile changes rapidly with time.

The purpose of this work was to determine the relationships between the experimental variables in X-ray microradiography and the errors in the derived effective diffusion coefficient, $D$, applicable to small regions of a permeable solid. The description of one-dimensional diffusion in a bounded plane by Fick's 1st Law has been taken as an example to illustrate the derivation of an expression for $D$ in terms of X-ray absorption data and sample thickness (Sect. 2). With this expression, the continuous functions in Fick's 1st Law are evaluated from experimental measurements made at discrete intervals of time and distance; this introduces errors into the derived value of $D$. Two other sources of error are also inherent in the experimental method. Firstly, the experimental intensity measurements are subject to uncertainty due to photon counting statistics. Secondly, the dynamic nature of the diffusion process results in a change in projected mass during the period of observation (counting time) for a given intensity measurement.

Numerical modelling has been used to determine how the distribution of the error in $D$ varies with typical experimental conditions. First of all, concentration profiles for an exemplary system were calculated (Sect. 3) using the explicit finite difference method [12]. Values of $D$, determined from these profiles sampled at intervals, were then used to assess the magnitude of errors in $D$ due only to sampling at finite positions and times (Sect. 4.1). Finally, simulated count data derived from the profiles were used to assess the additional effects of finite counts (Sect. 4.2) and changes in concentration during sample point measurement (Sect. 4.3). From these results, guidelines have been developed for optimization of the experimental parameters.

\section{Measurement of Diffusion Coefficient - Theoretical Formulation}

2.1 Application of Fick's 1st LaW to Measurement of One-Dimensional Diffusion IN A Bounded Plane. - Consider one-dimensional diffusion in the $x$ direction within the interconnecting fluid-filled pores of a porous solid. The solid phase is impermeable to the diffusing species. The twr -phase body is taken to be a rectangular parallelepiped of length $A$ in the $x$ direction, thickne s $B$ in the $z$ direction, and bounded by an impermeable plane at $x=A$ (Fig. 1). 


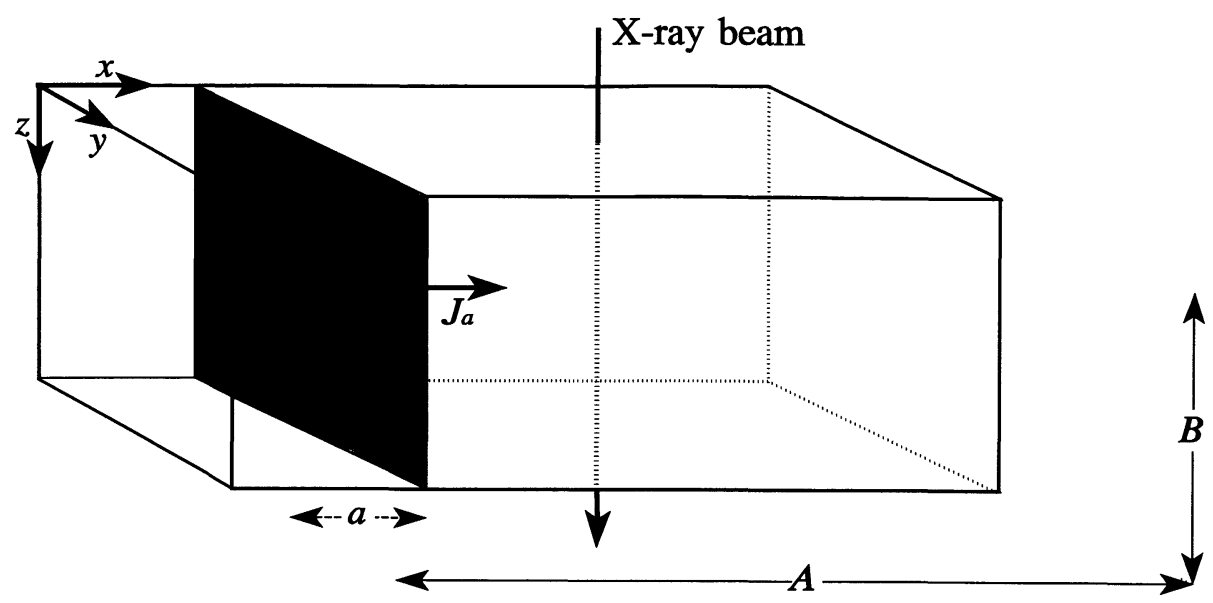

Fig. 1. - Schematic of the dimensions and coordinate system for one-dimensional diffusion in a permeable solid.

The fractional volume porosity, $\varepsilon_{x}$, is constant in the $y$ and $z$ directions but varies continuously in the $x$ direction. Thus $B_{x}^{\prime}$, the length of fluid traversed by the $\mathrm{X}$-ray beam in the $z$ direction, also varies with $x$. In order that the diffusion process, as characterised by $\mathrm{X}$-ray absorption measurements within this system, can be described by a continuous model, it must be assumed that the system can be regarded as homogeneous on a scale somewhat smaller that the diameter of the $\mathrm{X}$-ray beam used to make the measurements.

During diffusion at a constant temperature, and where $t$ is time: the concentration of diffusing species within the fluid,

$$
C=f(x, t),
$$

the flux through a unit area of the $y z$ plane at $0 \leq x<A$,

$$
J=f(x, t),
$$

and, in general, the effective diffusion coefficient

$$
D=f(x, C) .
$$

Assuming Fick's 1st Law is obeyed:

$$
J=-D\left(\frac{\partial C}{\partial x}\right)_{t}
$$

For the point, $x=a$, where $0<a<A$, the flux $J_{a}$ through a unit area of the $y z$ plane will equal the increase in mass per unit time of the diffusing species in the specimen between $x=a$ and $x=A$, divided by the cross-sectional area (Fig. 1). Thus:

$$
J_{a}=\int_{a}^{A}\left(\frac{\partial M}{\partial t}\right)_{x} \mathrm{~d} x=\frac{1}{B} \int_{a}^{A}\left(\frac{\partial m}{\partial t}\right)_{x} \mathrm{~d} x
$$

where $M$ is the mass of diffusing species per unit volume of the permeable solid, $m$ the mass of diffusing species per unit area projected onto the $x y$ plane at $x$, and $B$ the specimen thickness. 
$M$ and $m$ are both functions of $x$ and $t . B$ can be measured directly. $D$ can then be found from equations (1) and (2) provided that $(\partial C / \partial x)_{t}$ can be determined for $x=a$ and $(\partial m / \partial t)_{x}$ measured for all points between $x=a$ and $x=A$. The way in which this can be done is discussed in the next section.

2.2 Determination of Flux and Concentration Gradient From X-Ray Absorption DATA. - For a beam of monochromatic X-ray radiation in the $z$-direction (Fig. 1), the absorption due to the solid and fluid are independent, so that:

$$
I=I_{0} \exp -\left(\mu_{s} m_{s}+\mu_{\ell} m_{\ell}+\mu_{d} m_{d}\right)
$$

where $I_{0}$ (assumed to be constant throughout the experiments) and $I$ are the incident and transmitted intensities respectively, $\mu$ is the mass absorption coefficient, and subscripts $(s, \ell$ and $d$ ) refer to the solid, liquid (solvent) and diffusing species (the solute) respectively.

In a model experiment, $I$ is measured simultaneously at equispaced intervals of $\Delta x$ from $x=$ 0 to $x=A$. These measurements are repeated at equal time intervals $\Delta t$. A general point measured on this distance and time grid has coordinates $(p \Delta x, q \Delta t)$, where $p$ and $q$ are integers. The projected mass of the diffusing species, transmitted intensity and concentration at this point are denoted by $m_{p, q}, I_{p, q}$ and $C_{p, q}$ respectively. $B_{p}^{\prime}$ is the length of fluid traversed by the X-ray beam.

2.2.1 Determination of Flux. - Provided that $\mu_{\ell}$ is small, then during the time $(q-1) \Delta t$ to $(q+1) \Delta t$ over which the flux is measured, the change in $\mu_{\ell} m_{\ell}$, due to the change in volume occupied by the diffusing species, can be neglected. Thus from equation (3), the change in projected mass is given by:

$$
m_{p, q+1}-m_{p, q-1}=\frac{\ln I_{p, q-1}-\ln I_{p, q+1}^{\prime}}{\mu_{d}}
$$

so that

$$
\left(\frac{\partial m_{p, q}}{\partial t}\right)_{x} \approx \frac{\ln I_{p, q-1}-\ln I_{p, q+1}}{2 \Delta t \mu_{d}}
$$

Substituting into equation (2):

$$
J_{a}=\frac{1}{2 B \Delta t \mu_{d}} \int_{a}^{A}\left(\ln I_{p, q-1}-\ln I_{p, q+1}\right) \mathrm{d} x
$$

This integral can be evaluated using Simpson's rule. Thus provided $B$ is known, the flux can be determined.

2.2.2 Determination of Concentration Gradient. - Two approaches to determining the concentration gradient will be described. Both require knowledge of the values of $B_{p-1}^{\prime}$ and $B_{p+1}^{\prime}$. These can be determined from $I$ when the pores are filled by one and then another fluid (denoted by subscripts $f$ and $F$ ) with substantially different and known linear absorption coefficients $\mu_{f} \rho_{f}$ and $\mu_{F} \rho_{F}$, where $\rho_{f}$ and $\rho_{F}$ are the respective densities. From equation (3):

$$
B^{\prime}=\frac{\ln \left(I_{f} / I_{F}\right)}{\mu_{F} \rho_{F}-\mu_{f} \rho_{f}}
$$

If possible, fluid $f$ should be air, when $\mu_{f} \rho_{f}$ will be negligible. 
The first method for the determination of the concentration gradient follows from the expression:

$$
\left(\frac{\partial C_{p, q}}{\partial x}\right)_{t} \approx \frac{C_{p+1, q}-C_{p-1, q}}{2 \Delta x}
$$

Taking fluid $F$ as the solution of varying concentration during diffusion, $\left(\mu_{F} \rho_{F}\right)_{p-1, q}$ and $\left(\mu_{F} \rho_{F}\right)_{p+1, q}$ can be calculated from the respective $I_{F}$ values and the values of $B_{p-1}^{\prime}$ and $B_{p+1}^{\prime}$ determined from equation (7). Provided that the relation between $\mu_{F} \rho_{F}$ and the concentration $C$ of the diffusing species $d$ has been found in separate experiments, the concentration gradient can be determined.

An alternative approach is to calculate the change in concentration from the difference between the projected mass of the diffusing species at $x=(p+1) \Delta x$ and $(p-1) \Delta x$. From equation (3):

$$
\frac{\left(I_{F}\right)_{p-1, q}}{\left(I_{\ell}\right)_{p-1, q}}=\exp -\left\{\left(\mu_{\ell} \Delta m_{\ell}\right)_{p-1, q}+\left(\mu_{d} m_{d}\right)_{p-1, q}\right\}
$$

where $\Delta m_{\ell}$ is the increase in projected mass of the solvent $\ell$ due to the presence of the diffusing species $d$. If it is assumed that between $p-1, q$ and $p+1, q$, the change in $\Delta m_{\ell}$ is sufficiently small to be neglected, then:

$$
\begin{aligned}
\frac{\left(I_{F}\right)_{p+1, q}\left(I_{\ell}\right)_{p-1, q}}{\left(I_{\ell}\right)_{p+1, q}\left(I_{F}\right)_{p-1, q}} & =\exp \left\{-\left(\mu_{d} m_{d}\right)_{p+1, q}-\left(\mu_{d} m_{d}\right)_{p-1, q}\right\} \\
& =\exp \left\{-\mu_{d} \Delta m_{d}\right\}
\end{aligned}
$$

Since this method allows only the determination of the increase in projected mass $\Delta m_{d}$, and not the individual values of $m_{d}$, correction must be made for the increase in $B^{\prime}$ between $(p-1)$ and $(p+1)$. As $m_{d}=C B^{\prime}$ and with the approximations that $B_{p-1}^{\prime}+B_{p+1}^{\prime}=2 B_{p}^{\prime}$ and $B_{p-1}^{\prime} \cdot B_{p+1}^{\prime}=$ $B_{p}^{\prime 2}$ :

$$
C_{p+1, q}-C_{p-1, q}=\frac{\Delta m_{d}-C_{p, q}\left(B_{p+1}^{\prime}-B_{p-1}^{\prime}\right)}{B^{\prime}{ }_{p}}
$$

$C_{p, q}$ must be determined from $\mu_{F} \rho_{F}$, derived via equation (7). Whether the required variation of $\mu_{F} \rho_{F}$ with $C$ can be determined sufficiently accurately from the mass absorption coefficients and estimated densities, instead of being determined in separate experiments, depends on the relative magnitude of the terms in the numerator of the right-hand side of equation (11).

2.2.3 The Special Case Where Porosity Does Not Vary With Distance. - When the volume porosity $\varepsilon$ is independent of $x$, equation (11) reduces to:

$$
C_{p+1, q}-C_{p-1, q}=\frac{\Delta m_{d}}{B^{\prime}}
$$

Substituting for $\Delta m_{d}$ from equation (10) gives:

$$
\frac{\partial C_{p, q}}{\partial x} \approx \frac{\ln I_{p-1, q}-\ln I_{p+1, q}}{2 B^{\prime} \Delta x \mu_{d}}
$$

Rearranging equation (1) and substituting from expressions (2), (5) and (13), gives:

$$
D \approx-\varepsilon \frac{\Delta x}{\Delta t} \frac{1}{\ln \left(I_{p-1, q} / I_{p+1, q}\right)} \int_{a}^{A} \ln \left(\frac{I_{p, q-1}}{I_{p, q+1}}\right) \mathrm{d} x
$$


where $B^{\prime} / B$ has been replaced by $\varepsilon$. Thus if $\varepsilon$ has been measured separately, $D$ can be found directly from intensity measurements without knowledge of $\mu_{d}$ or the relationship between $\mu_{F} \rho_{F}$ and $C$.

2.3 Errors Due to Counting Statistics. - If the X-ray intensity $I_{p, q}$ is measured by a photon counting system, the number of photons, $N_{p, q}$, detected in a fixed time $\tau$, has a Poisson distribution with standard deviation $\bar{N}_{p, q}^{1 / 2}$, where $\bar{N}_{p, q}$ is the mean count in time $\tau$. Thus the fractional error in $I_{p, q}$ is:

$$
\frac{\sigma\left(I_{p, q}\right)}{I_{p, q}}=\frac{\sigma\left(\bar{N}_{p, q}\right)}{\bar{N}_{p, q}}=\frac{1}{\sqrt{\bar{N}_{p, q}}}
$$

where $\sigma()$ indicates the standard deviation of the respective variable within the brackets. Therefore the effect of counting statistics on the error in $D$ can be estimated by combining the individual errors of the constituent measurements of intensities (taken as their standard deviations with the assumption that the measurements are uncorrelated) using the rules for combination of errors based on partial differentiation.

2.3.1 Calculation of Error in $J_{a}$. - Replacing the integral in equation (6) by a summation from $x=a$ to $x=A$ :

$$
J_{a} \approx \frac{\Delta x}{2 B \Delta t \mu_{d}} \sum \ln \frac{N_{p, q-1}}{N_{p, q+1}}
$$

So that:

$$
\left(\sigma\left(J_{a}\right)\right)^{2}=\left(\frac{\Delta x}{2 B \Delta t \mu_{d}}\right)^{2} \sum\left(\sigma\left(\ln \frac{N_{p, q-1}}{N_{p, q+1}}\right)\right)^{2} .
$$

Making the substitution that:

$$
\left(\sigma\left(\ln \frac{N_{p, q-1}}{N_{p, q+1}}\right)\right)^{2}=\frac{1}{N_{p, q-1}}+\frac{1}{N_{p, q+1}}
$$

gives:

$$
\left(\sigma\left(J_{a}\right)\right)^{2}=\left(\frac{\Delta x}{2 B \Delta t \mu_{d}}\right)^{2}\left(\sum \frac{1}{N_{p, q-1}}+\sum \frac{1}{N_{p, q+1}}\right) .
$$

Thus:

$$
\left(\frac{\sigma\left(J_{a}\right)}{J_{a}}\right)^{2}=\left(\sum \frac{1}{N_{p, q-1}}+\sum \frac{1}{N_{p, q+1}}\right) /\left(\sum \ln \frac{N_{p, q-1}}{N_{p, q+1}}\right)^{2}
$$

2.3.2 Calculation of Error in Concentration Gradient. - Replacing intensities by counts in equation (13) and differentiating gives:

$$
\sigma\left(\frac{\partial C_{p, q}}{\partial x}\right) \approx \frac{1}{2 B^{\prime} \Delta x \mu_{d}}\left(\frac{\sigma\left(N_{p-1, q} / N_{p+1, q}\right)}{N_{p-1, q} / N_{p+1, q}}\right)
$$




$$
=\frac{1}{2 B^{\prime} \Delta x \mu_{d}}\left(\frac{1}{N_{p-1, q}}+\frac{1}{N_{p+1, q}}\right)^{1 / 2}
$$

Thus:

$$
\sigma\left(\frac{\partial C_{p, q}}{\partial x}\right) / \frac{\partial C_{p, q}}{\partial x}=\left(\frac{1}{N_{p-1, q}}+\frac{1}{N_{p+1, q}}\right)^{1 / 2} / \ln \left(\frac{N_{p-1, q}}{N_{p+1, q}}\right)
$$

2.3.3 Calculation of Error in Effective Diffusion Coefficients. - From equations (20) and (23):

$$
\begin{aligned}
&\left(\frac{\sigma\left(D_{a}\right)}{D}\right)^{2}=\left(\sum\right.\left.\frac{1}{N_{p, q-1}}+\sum \frac{1}{N_{p, q+1}}\right) /\left(\sum \ln \frac{N_{p, q-1}}{N_{p, q+1}}\right)^{2} \\
&+\left(\frac{1}{N_{p-1, q}}+\frac{1}{N_{p+1, q}}\right) /\left(\ln \frac{N_{p-1, q}}{N_{p+1, q}}\right)^{2}
\end{aligned}
$$

The values of $\sigma\left(D_{a}\right) / D$ calculated from equation (24) are discussed in the following section when they are compared with errors determined from numerical simulations.

\section{Numerical Simulation of Concentrations During Diffusion}

Although the preceding equations show how diffusion coefficients can be determined for the general situation, the present analysis of the errors is restricted to the case in which $D$ and $B^{\prime}$ are cunstant in the $x$ direction. This restriction is reasonable because the analysis of errors will still give a good guide to the more general case, provided $D$ and $B^{\prime}$ do not vary too rapidly with $x$.

For a permeable solid with constant porosity (as shown in Fig. 1), Fickian diffusion within the liquid phase is described by:

$$
\frac{\partial C}{\partial t}=\frac{D}{\varepsilon} \frac{\partial^{2} C}{\partial x^{2}}=D^{*} \frac{\partial^{2} C}{\partial x^{2}}
$$

Using the dimensionless variables:

$$
X=x / A, \quad T=D^{*} t / A^{2}, \quad c=C / C_{0}
$$

where $C_{0}$ is a constant concentration, equation (25) becomes:

$$
\frac{\partial c}{\partial T}=\frac{\partial^{2} c}{\partial X^{2}}
$$

Ideal concentration profiles during diffusion were calculated using the explicit finite-difference method [12] with boundary conditions:

$$
\begin{array}{lll}
c=0, & 0 \leq X \leq 1, & T=0, \\
c=1, & X=0, & T>0, \\
c=f(X, T), & 0<X \leq 1, & T>0 .
\end{array}
$$

The range of $X$ and of time are divided into equispaced intervals $\delta X$ and $\delta T$ respectively, so that the coordinates of a representative node on the rectangular grid can be denoted by $(i \delta X, j \delta T)$, where $i$ and $j$ are integers. The value of $c$ at this point is denoted $c_{i, j}$. Then: 


$$
c_{i, j+1}=c_{i, j}+\frac{\delta T}{(\delta X)^{2}}\left(c_{i-1, j}-2 c_{\imath, j}+c_{i+1, j}\right)
$$

A set of dimensionless concentration data was calculated, with $\delta X=1 \times 10^{-3}$ and $\delta T=4 \times 10^{-7}$, which conformed to the constraint required for a stable solution [13]:

$$
0<\frac{\delta T}{(\delta X)^{2}} \leq \frac{1}{2}
$$

These particular values of $\delta X$ and $\delta T$ were chosen so that the dimensionless concentration data could be transformed to dimensioned data using values for the effective diffusion coefficient, fractional volume porosity, distance and time intervals within the range corresponding with a previous experiment [11]. The transformed set of concentration data thus included calculated profiles that matched experiment without any need for interpolation.

\section{Assessment of Errors in Diffusion Coefficients}

4.1 Effects of Sampling at Finite Distances and Times. - At infinite count rate, there would be no uncertainty in $D$ from counting statistics or changes in concentration during observation. Nevertheless, there would be an error in each calculated value of $D$ because the continuous functions (Eqs. (1) and (2)) are only approximated by the use of data at finite distance and time intervals, $\Delta x$ and $\Delta t$.

Values of $D$ were calculated from simulated concentration data. Sampling intervals were $\Delta X \gg$ $\delta X$ and $\Delta T \gg \delta T$ in order to reduce the effect of errors in the simulated data due to truncation of the Taylor series implicit in equation (27). The concentration gradient was found from equation (8) and the flux was found by the method in section 2 , but using:

$$
m_{p, q+1}-m_{p, q-1}=B^{\prime}\left(C_{p, q+1}-C_{p, q-1}\right)
$$

instead of equation (4). Figure 2 illustrates, on an $X: T$ grid, contour lines of constant fractional error in $D$. For the four combinations of $\Delta X$ and $\Delta T$ investigated, there was a very extensive region within which the fractional error was less than 0.005 and varied only slowly with $X$ and $T$. The extent of the $(X, T)$ regions in which there were very large positive errors $(X \rightarrow 1, T \rightarrow 0)$ or large negative errors $(X \approx 0.2, T \rightarrow 0)$ varied with the $\Delta X, \Delta T$ combination.

These calculations show that there should normally be no difficulty in choosing a combination of $\Delta X$ and $\Delta T$ so that fractional errors in the calculated value of $D$, due to finite sampling, are less than 0.005 for all but extreme values of $X$ and $T$.

\subsection{Effects of Counting Statistics.}

4.2.1 Distribution of Effective Diffusion Coefficients Determined by Monte Carlo Method. - As explained earlier, $N_{p, q}$ has a random distribution, so that, for each $(p \Delta x, q \Delta t)$, X-ray measurements in repeated experiments under identical conditions would yield a distribution of values of the calculated effective diffusion coefficient, $D_{p, q}^{\prime}$.

The effect of finite observation time was neglected so that concentrations were assumed to remain constant during X-ray counting. Dimensionless data were transformed to simulate concentrations of diffusing species in the solvent for typical experimental conditions (Tab. I). Examples of the concentration-distance profiles are shown in Figure 3. The concentration data were then converted to 4000 sets of simulated count data conforming to Poisson statistics for each order 
Table I. - Sample and experimental parameters used in the transformation of simulated dimensionless diffusion data.

\begin{tabular}{|c|c|c|}
\hline Specimen & $\begin{array}{l}A \text { (length) } \\
B \text { (thickness) } \\
\varepsilon \text { (fractional volume } \\
\text { porosity) }\end{array}$ & $\begin{array}{l}1.0 \mathrm{~cm} \\
0.2 \mathrm{~cm} \\
0.25\end{array}$ \\
\hline Diffusant (KI) & $\begin{array}{l}D \text { (effective } \\
\text { diffusion } \\
\text { coefficient) } \\
\mu_{d} \text { (mass absorption } \\
\text { coefficient for Mo } \\
\left.K_{\alpha}\right)\end{array}$ & $\begin{array}{l}2.5 \times 10^{-6} \mathrm{~cm}^{2} \mathrm{~s}^{-1} \\
32.08 \mathrm{~g}^{-1} \mathrm{~cm}^{2}\end{array}$ \\
\hline $\begin{array}{l}\text { Experimental } \\
\text { parameters }\end{array}$ & $\begin{array}{l}C_{0} \text { (external } \\
\text { concentration) } \\
\Delta x \text { (distance } \\
\text { interval) } \\
\Delta t \text { (time interval) }\end{array}$ & 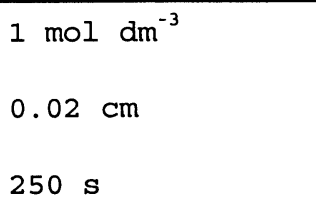 \\
\hline
\end{tabular}

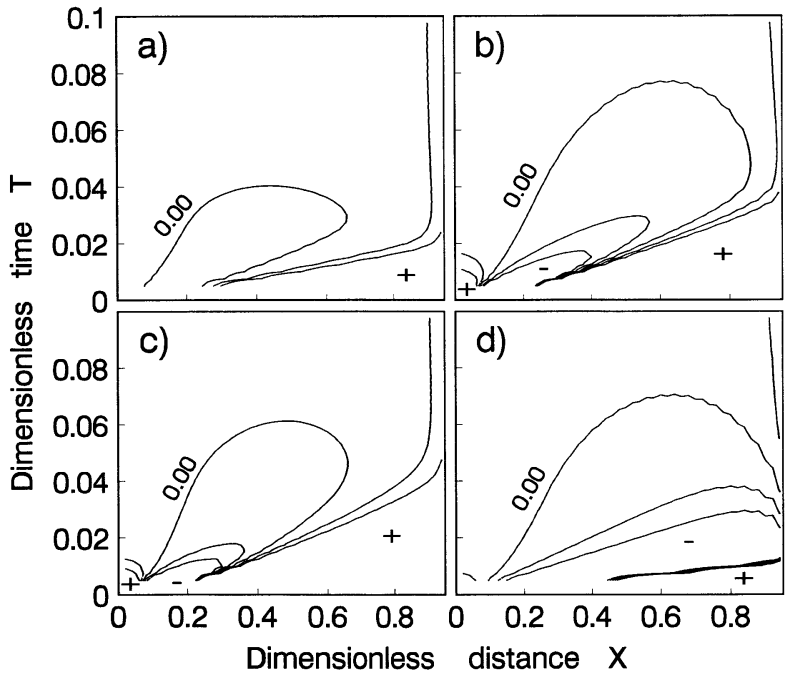

Fig. 2. - Contours of constant fractional error in $D$ (at 0.005 intervals in the range -0.01 to +0.01 ) as a function of $T$ and $X$. Areas in which fractional error $<-0.01$ are marked - , and areas in which fractional error $>0.01$ are marked +. a) $\Delta X=0.004, \Delta T=0.0005$, b) $\Delta X=0.020, \Delta T=0.0025$, c) $\Delta X=0.004$, $\Delta T=0.0025, \mathrm{~d}) \Delta X=0.020, \Delta T=0.0005$.

of magnitude in the range from $10^{4}$ to $10^{9}$ of $\bar{N}_{0}$, the mean number of detected photons at each point passing through the sample in the absence of diffusant. Additionally, the calculation was performed without randomization to produce the set of 'noise-free' count data (effectively the 


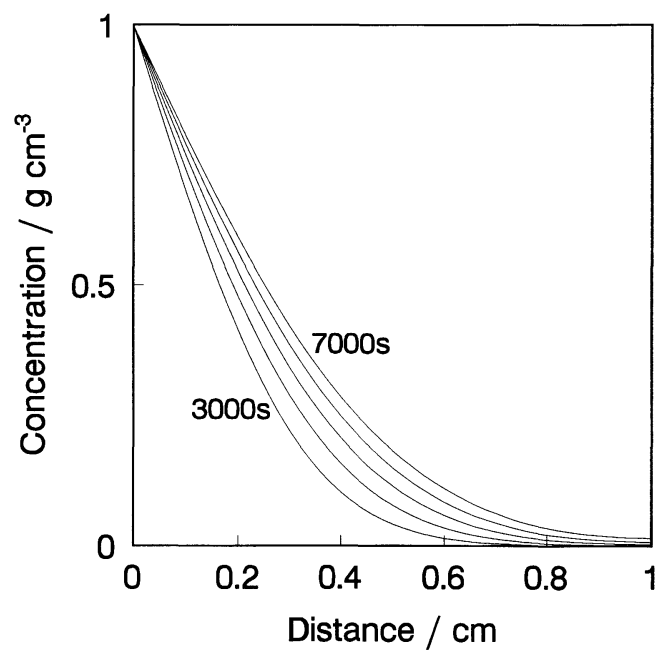

Fig. 3. - Examples of simulated concentration profiles: 3000 to $7000 \mathrm{~s}$ at $1000 \mathrm{~s}$ intervals.

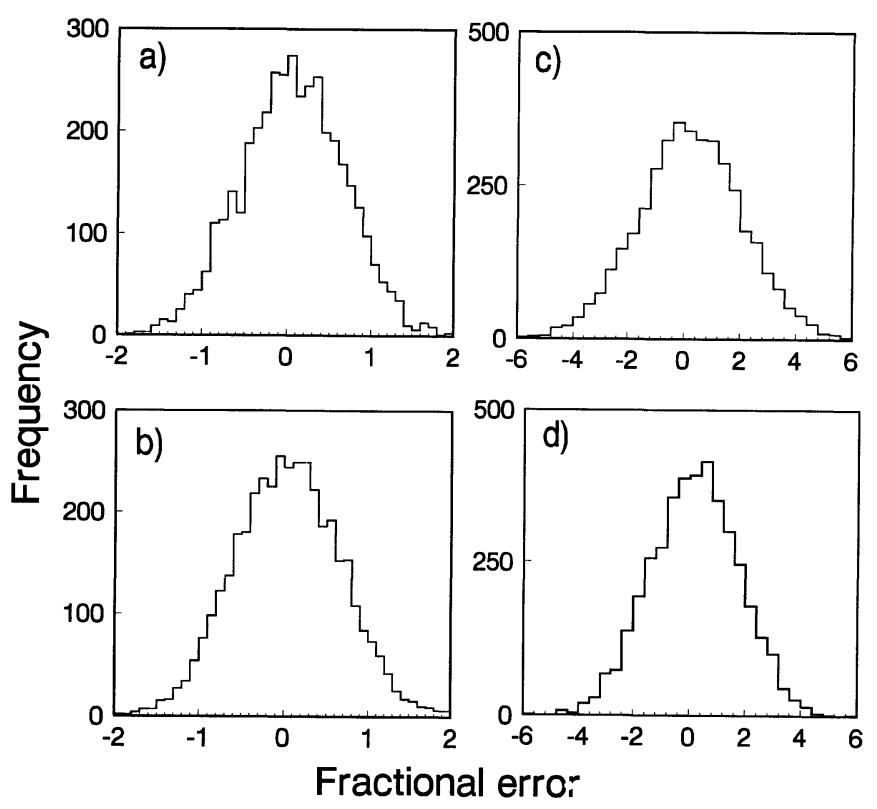

Fig. 4. - Examples of distributions calculated from simulated count data at $5000 \mathrm{~s}$ diffusion time for $\bar{N}_{0}=$ $10^{4}$. Concentration gradient a) and flux b) at $0.1 \mathrm{~cm}$; concentration gradient c) and flux d) at $0.5 \mathrm{~cm}$.

mean count values for infinite counting time) for each value of $\bar{N}_{0}$.

The simulated count data were used to investigate the distributions, including standard deviations, of the concentration gradient, flux and $D_{p, q}^{\prime}$ at distances from 0.1 to $0.8 \mathrm{~cm}$ and at times from 3000 to $7000 \mathrm{~s}$. The standard deviations were also calculated from the formulae in Section 2.3 applied to the 'noise-free' count data. For both these calculations, Figure $2 \mathrm{~b}$ shows that the approximations due to the finite sampling intervals lead to a fractional error in $D$ of less than 

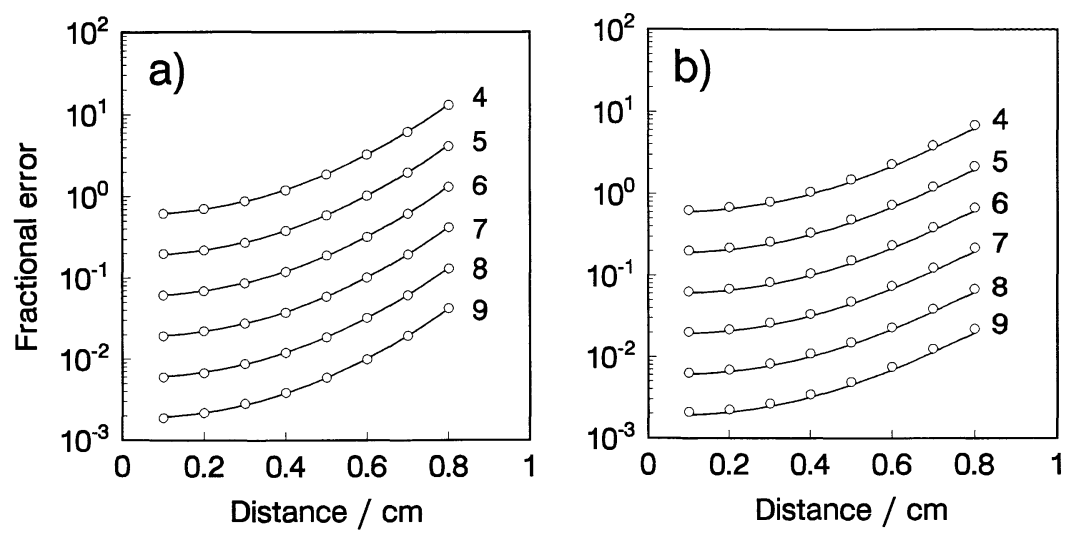

Fig. 5. - Variation of fractional error in concentration gradient a) and flux b), with distance at $5000 \mathrm{~s}$, determined analytically ( - ) and by Monte Carlo calculations $(O)$ for $\bar{N}_{0}$ from $10^{4}$ to $10^{9}$ (lines labelled with exponent).

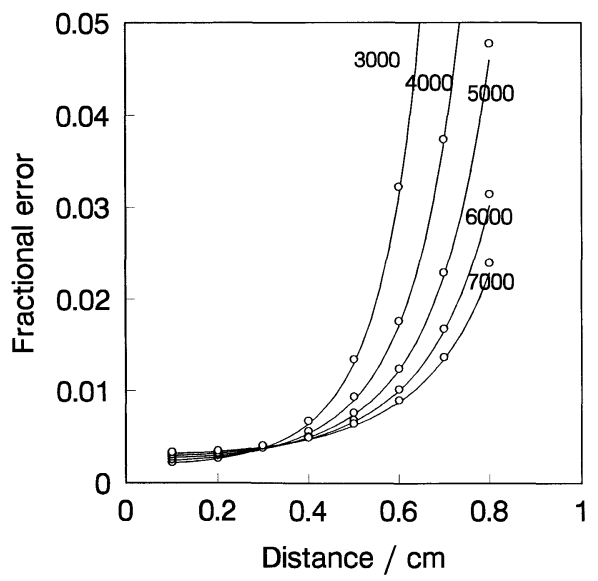

Fig. 6. - Variation of fractional error in $D$ with distance at $1000 \mathrm{~s}$ intervals, determined analytically ( - ) and by Monte Carlo calculations $(O)$ for $\bar{N}_{0}=10^{9}$ (lines labelled with diffusion times (s)).

0.005 .

Even at the lowest value of $\bar{N}_{0}$ that was investigated $\left(10^{4}\right)$, the Monte Carlo distributions of concentration gradients and flux were symmetrical (Fig. 4), with sample standard deviations in good agreement with the values derived analytically (Fig. 5). For a given combination of position and time, an approximately $n^{2}$-fold increase in $\bar{N}_{0}$ gave approximately $n$-fold reductions in both standard deviations, as expected from Equations (19) and (22).

For a given $\bar{N}_{0}$, with increasing distance $(0.1$ to $0.8 \mathrm{~cm})$ at a fixed time, the standard deviation in concentration gradient decreased $\sim 10 \%$ and the standard deviation in flux decreased $\sim 55 \%$. However, again for a given $\bar{N}_{0}$, with increasing time (3000 to 7000 s) at a fixed distance, both standard deviations increased $\sim 5 \%$. By contrast, the decrease in the absolute values of concentration gradient and flux with increasing distance has a profound effect on the sizes of the fractional errors: for example at $3000 \mathrm{~s}$ and between 0.1 and $0.8 \mathrm{~cm}$, the fractional error in concentration gradient increased $\sim 150$-fold and the fractional error in flux increased $\sim 60$-fold. At $0.1 \mathrm{~cm}$ the 


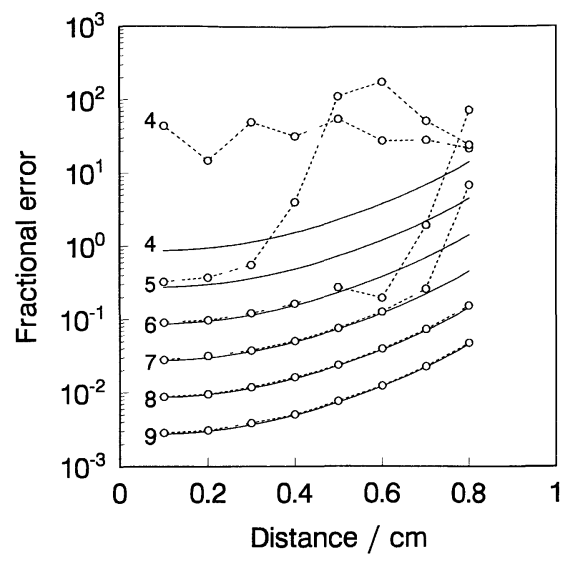

Fig. 7. - Variation of fractional error in $D$ with distance at $5000 \mathrm{~s}$ determined analytically ( - ) and by Monte Carlo calculations $(\cdots \bigcirc \cdots)$ for $\bar{N}_{0}$ from $10^{4}$ to $10^{9}$ (lines labelled with exponent).

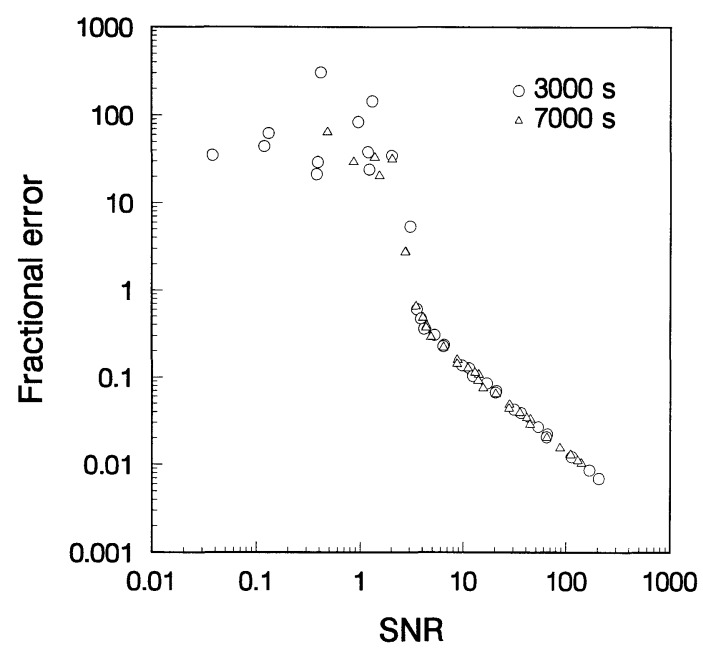

Fig. 8. - Variation of observed fractional error in $D$ with $S N R$ for concentration gradient data at $3000 \mathrm{~s}$ and $7000 \mathrm{~s}$, for distances from 0.1 to $0.8 \mathrm{~cm}$ at $0.1 \mathrm{~cm}$ intervals, and $\bar{N}_{0}$ from $10^{4}$ to $10^{8}$.

fractional error in the flux exceeded that in the concentration gradient by $\sim 5 \%$, but by $0.3 \mathrm{~cm}$ the fractional error in concentration gradient was predominant. The combined effects of these errors on the fractional error in $D_{p, q}^{\prime}$ is discussed in the following paragraph.

At high values of $\bar{N}_{0}$, within the range investigated, the distributions of $D_{p, q}^{\prime}$ were symmetrical; but with decreasing $\bar{N}_{0}$, the distributions became increasingly skewed and had more extreme outliers. Figure 6 shows that for $\bar{N}_{0}=10^{9}$, there was good agreement between the values of the fractional error in $D_{p, q}^{\prime}$ observed for the Monte Carlo calculations and those predicted analytically. However as $\bar{N}_{0}$ decreased (Fig. 7, note its logarithmic vertical scale), the point at which 


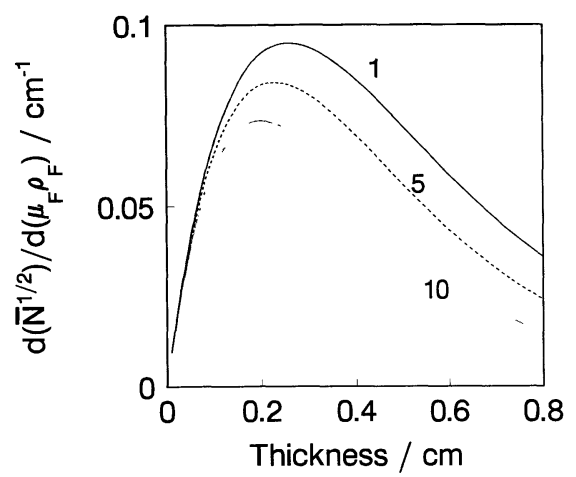

Fig. 9. - Variation of sensitivity to change in $\mu_{F} \rho_{F}, \mathrm{~d}\left(\bar{N}^{1 / 2}\right) / \mathrm{d}\left(\mu_{F} \rho_{F}\right)$, as a function of thickness $B$ for $\mu_{s} \rho_{s}=10 \mathrm{~cm}^{-1}, \varepsilon=0.25$ and $\mu_{F} \rho_{F}=1 \mathrm{~cm}^{-1}(-), 5 \mathrm{~cm}^{-1}(--)$, and $10 \mathrm{~cm}^{-1}(\cdots)$.

agreement between predicted and observed errors broke down moved to smaller distances.

The origin of the breakdown of the analytical prediction was sought in the statistical uncertainty of the count data. The determinations of concentration gradient and flux both depend on the measurement of the small differences between counts collected at next-nearest-neighbour distances or times. In general, the confidence interval for the signal, i.e. the difference between the counts $\left(N_{1}-N_{2}\right)$, is determined by the noise $\left(N_{1}+N_{2}\right)^{1 / 2}$. From the preceding investigation of the variation in fractional errors for concentration gradient and flux, it would be expected that the error in $D_{p, q}^{\prime}$ would be more strongly affected by the concentration gradient count data. For the mean count $\bar{N}_{0}$, the signal-to-noise ratio for concentration gradient count data, $S N R$ :

$$
S N R=\frac{\bar{N}_{p+1, q}-\bar{N}_{p-1, q}}{\left(\bar{N}_{p+1, q}+\bar{N}_{p-1, q}\right)^{\frac{1}{2}}}
$$

Figure 8 shows that for $S N R<\sim 3$, the observed fractional error in $D$ increases very rapidly. For $S N R>\sim 3$, there is an inverse relationship between $S N R$ and the observed fractional error in $D$, so that:

$$
\frac{\bar{N}_{p+1, q}-\bar{N}_{p-1, q}}{\left(\bar{N}_{p+1, q}+\bar{N}_{p-1, q}\right)^{\frac{1}{2}}}=\frac{D}{\sigma(D)}
$$

These calculations show that the mean count $\bar{N}_{0}$, and hence the counting time, required to determine $D$ within a specific confidence interval can be found from estimates of $S N R$ for concentration gradient data at the particular distance and time.

4.2.2 Optimum Thickness of Sample, B. - We now address the problem of the optimum value of $B$ for determination of changes in concentration of the diffusing species. In general, the signalto-noise ratio of the mean number of detected photons passing through the sample is $\bar{N} / \bar{N}^{\frac{1}{2}}$, i.e. $\bar{N}^{\frac{1}{2}}$. From equation (3) and consideration of the relative contributions of solid and fluid to the pathlength in the sample, it follows that:

$$
\bar{N}^{\frac{1}{2}}={\overline{N_{0}}}^{* \frac{1}{2}} \exp \left\{\frac{-\mu_{s} \rho_{s}(1-\varepsilon) B-\mu_{F} \rho_{F} \varepsilon B}{2}\right\}
$$


where $\bar{N}_{0}^{*}$ is the mean number of incident photons. The optimum sample thickness is that for which the sensitivity to change in $\mu_{F} \rho_{F}$, i.e. $\mathrm{d}\left(\bar{N}^{1 / 2}\right) / \mathrm{d}\left(\mu_{F} \rho_{F}\right)$, is a maximum with respect to $B$. It can be shown by differentiation of equation (32) that this condition is met when:

$$
B\left\{\mu_{s} \rho_{s}(1-\varepsilon)+\mu_{F} \rho_{F} \varepsilon\right\}=2
$$

Since the value of $\mu_{F} \rho_{F}$ varies with time and position during diffusion, there is no single optimum value for $B$. However Figure 9 shows that, in this example, the effect of the variation in $\mu_{F} \rho_{F}$, within the range 1 to $10 \mathrm{~cm}^{-1}$, is negligible for the practical purpose of choosing the optimum sample thickness.

4.3 Effect of Finite Counting Time. - During a finite counting time, the concentration of diffusing species in the beampath changes as diffusion proceeds. Further, the relationship between the transmitted intensity and concentration is nonlinear (Eq. 3), so the measured count rate at a given position does not represent the projected mass of diffusing species at the midpoint of the counting time. Nevertheless, for the system studied, the relationship between $I_{0} / I$ and $t$ was close to linear over extended times. In the ranges investigated (Sect. 4.2), the difference in mean counts for the average for measurements at 251 consecutive $1 \mathrm{~s}$ times and the measurement at the midpoint of the $251 \mathrm{~s}$ was less then $0.002 \%$.

\section{Discussion}

Three sources of error have been discussed: the use of finite sampling intervals for distance and time, uncertainty related to counting statistics, and the finite time for measurement of a dynamic process. The use of finite measurements leads to a reduction in the accuracy with which $D$ can be determined. However, in many cases, these effects will be negligible in comparison with the effect of statistical uncertainty in count rate on the precision with which $D$ can be determined. For example, in the typical example investigated, the very small reduction in accuracy with increased counting time was negligible in comparison with the improvement in precision resulting from the increased photon counts. Likewise, the use of finite sampling intervals gave rise to very small errors, except for extremes of distance and time of measurement which could be excluded in experimental design. For the experimental parameters in Table I and a transmitted count $1 \times 10^{5}$, the fractional error is $\sim 0.4$ at distances from 0.1 to $0.3 \mathrm{~cm}$. This magnitude of error is generally acceptable and is consistent with the experimental work on the determination of $D$ in a glass frit [11].

Analysis of the errors due to finite sampling intervals shows that the method is unsuitable for determinations at great depth $(x / A)$ within the sample or at very early times during the diffusion process. These extreme conditions, which give rise to large errors, are associated with extremes of concentration gradient and flux and can be identified by inspection of concentration: distance profiles (Fig. 3), calculated from an estimate of $D$, or obtained experimentally. For less extreme conditions, the error due to finite sampling can be calculated for each position and time using an experimentally derived estimate of $D$. This can then be used to improve the estimate.

When a laboratory $\mathrm{X}$-ray source is used, and particularly when there is substantial attenuation by the solid phase, there may be overwhelming uncertainty in the estimates of $D_{p, q}^{\prime}$ arising from photon counting statistics (see for example Fig. 7). We now consider strategies for reducing these errors.

If, for a given system, the change in transmitted intensity, $I_{p, q}$, with time can be taken as linear, $\bar{N}_{0}$ can be increased by prolonging the counting time to equal the duration of the time sampling interval $\Delta t$ and taking the cumulative counts as the mid-time value. With a typical laboratory 
source [11], extending counting time to 250 s would give $\bar{N}_{0}=\sim 3.5 \times 10^{6}$. For the example considered above, this would permit estimation of $D_{p, q}^{\prime}$ with a fractional error of $<0.1$ for distances of 0.1 to $0.3 \mathrm{~cm}$. This method can only be used with a linear array detector. If a single detector is used, the counting time at each position is limited and interpolation must be used to refer all measurements to the same time during diffusion. In this case, it is probable that errors can be reduced by counting strategies in which, for example, the counting time varies with position.

The statistical error can also be reduced by optimizing the sample thickness. The optimum range of thickness can be calculated, given the porosity of the solid and the linear absorption coefficients of the solid, solvent and solution (with concentration $C_{0}$ ) for the relevant X-ray energy. For given conditions, the precision is also improved by increasing $C_{0}$ and/or $\mu_{d}$.

For a constant counting time, precision improves with increase in $\Delta t$ or in the distance interval used for determination of the concentration gradient, or with a reduction in distance intervals used for determination of flux. However, care must be taken that an improved precision in $D^{\prime}$ due to a change in sampling intervals is not unduly offset by a loss in accuracy, so a preliminary assessment of these errors (as in Sect. 2.1) is necessary.

An improved estimate of $D_{x}^{\prime}$ can be obtained by combining alternate pairs of estimates of $D_{q}^{\prime}$ (where $q=2 n, 2(n+1)$ ) at the chosen position. This selection avoids introducing bias due to correlation between the estimates of $D_{q}^{\prime}$ for $(q=n)$ and $(q=n+1)$ arising from the use of the same intensity measurements in the calculation of flux.

A rather different approach to reducing the errors due to photon counting statistics is to set up steady periodic concentrations within the medium so that repeated measurements can be made under identical conditions. In such a system, the concentration at $x=0$ is varied as a square wave between $C_{0}$ and 0 . If $D$ is constant or a function of position only, then the cycle of diffusion in and out rapidly tends to a steady cyclical pattern of concentrations with the same frequency as the surface oscillation. This result is based on the analogous theory of the conduction of heat in solids [15]. When the pattern is established (typically after six cycles), counts can be summed for corresponding measurements at the same distances and times within a cycle, over as many cycles as are needed to reduce the statistical uncertainty by the desired amount.

We now consider ways in which errors due to counting statistics can be reduced by instrumentation. One method is to increase the X-ray flux by using a different source e.g. a rotating anode tube or synchrotron, or with a loss in spatial resolution, by increasing the aperture size. However, an increased flux will very rapidly introduce unacceptable nonlinearities and eventual saturation in a photon counting system. This could probably be overcome if the photon counter were replaced by another system such as a scintillation detector and photomultiplier used in current mode. However, attention would have to be paid to the stability of such a system as small changes have to be accurately measured within and between scans.

In considering the general application of this method, based on Fick's 1st Law, there are two conditions that have to be met. The first is that the surface through which the flux is being measured and which contains the point at which the diffusion coefficient is to be determined should lie in a surface which encloses a known volume that is entirely accessible for the measurement of changes in the diffusant concentration. The second condition is that the flux should be constant over a known area of the surface and zero everywhere else. This means that 1-dimensional diffusion can only be studied in a system with a bounding plane. In 2-dimensions, these conditions cannot be met. In 3-dimensions, in which the concentrations could be measured by microtomographic methods, there must be spherical symmetry. If the system under study does not conform to these restrictions, the local diffusion coefficient must be determined from measurements made entirely locally. This can be done using a method based on Fick's 2 nd Law in which $D$ is determined from local measurements of $\partial C / \partial t$ and $\partial^{2} C / \partial x^{2}[14]$.

The useful application of X-ray quantum counting methods to diffusion studies is potentially 
restricted by lack of precision in the measurements as a consequence of counting statistics. High intensity X-ray sources, in absorption and in fluorescence methods, can yield results with high precision even for species at low concentration. XRF also allows straightforward elemental discrimination so that, for example, multicomponent diffusion can be followed [3]. The disadvantage of high intensity sources is their very limited availability. This work and a previous experimental study [11] have shown that useful results with absorption methods can be obtained by use of a relatively weak laboratory source, provided that attention is given to optimization of experimental conditions. Although the guidelines have been developed with reference to a very simple system, the principles could be extended to more complex ones. For example, there is the potential to study 3- dimensional diffusion by use of X-ray microtomography. Additionally, there is the potential to study ternary diffusion since elemental discrimination can be obtained with a laboratory $\mathrm{X}$-ray source, by use of a multichannel analyser to measure absorption on either side of the element absorption edges [16]. A detailed consideration of another approach has been given in which the concentration of $n$ elements is determined from the absorption at $n$ different energies [17].

\section{Acknowledgements}

We thank Dr. G.H. Dibdin for helpful discussions at an early stage of this work. This work was supported in part by the Special Trustees of The Royal London Hospital.

\section{References}

[1] Tanigaki M., Kondo K., Harada M. and Eguchi W., J. Phys. Chem. 87 (1983) 586.

[2] Eguchi W., Harada M., Adachi M., Tanigaki M. and Kondo K., J. Chem. Eng. Jpn. 17 (1984) 472.

[3] Anderson P., Elliott J.C., Thomas C.R. and Van Langevelde F., X-ray Spectrom. 22 (1993) 265.

[4] Kärger J. and Ruthven D.M., Diffusion in Zeolites and other Microporous Solids (John Wiley \& Sons, New York, 1992).

[5] Dibdin G.H., Elliott J.C. and Anderson P., Mater. Res. 2 (1987) 178.

[6] Darling A.I., Mortimer K.V., Poole D.F.G. and Ollis W.D., Arch. Oral. Biol. 5 (1961) 251.

[7] Timofeev D.P. and Voskresenskii A.A., Dolk. Akad. Nauk. SSSR 122 (1958) 434.

[8] Voloshchuk A.M. and Dubinin M.M., Dolk. Akad. Nauk. SSSR 212 (1973) 649.

[9] Dubinin M.M., Erashko I.T., Kadlec O., Ulin V.I., Voloshchuk A.M. and Zolotarev P.P., Carbon 13 (1975) 193.

[10] Rondot S., Cazaux J., Aaboubi O., Chopart J.P. and Olivier A., Science 263 (1994) 1739.

[11] Anderson P., Dowker S.E.P., Elliott J.C. and Dibdin G.H., in X-ray Microsc. III. A.G. Michette, G.R. Morrison and C.J. Buckley Eds. (Springer-Verlag, Berlin, Heidelberg) Springer Series Opt. Sci. 67 (1992) 435.

[12] Crank J., The Mathematics of Diffusion (2nd Edition, Oxford University Press, Oxford, 1975).

[13] Smith G.D., Numerical Solution of Partial Differential Equations (Oxford University Press, Oxford, 1965).

[14] Anderson P., Dowker S.E.P. and Elliott J.C., in preparation.

[15] Carslaw H.S. and Jaeger J.C., Conduction of Heat in Solids (2nd Edition, Clarendon Press, Oxford, 1959).

[16] Anderson P., Davis G.R. and Elliott J.C., Microscopy and Analysis (March 1994) 31.

[17] Cazaux J., Microsc. Microanal. Microstruct. 4 (1993) 513. 


\section{Nomenclature}

\begin{tabular}{|c|c|}
\hline$a$ & general position, $0<a<A[\mathrm{~cm}]$ \\
\hline$A$ & position of impermeable plane on $x$-axis [cm] \\
\hline$B$ & constant thickness of solid in $z$ direction [cm] \\
\hline$B^{\prime}$ & $\mathrm{X}$-ray pathlength within pores $[\mathrm{cm}]$ \\
\hline$c$ & dimensionless variable, $C / C_{0}$ \\
\hline$C$ & concentration of diffusing species within fluid $\left[\mathrm{g} \mathrm{cm}^{-3}\right]$ \\
\hline$C_{0}$ & constant concentration at $x=0, t>0\left[\mathrm{~g} \mathrm{~cm}^{-3}\right]$ \\
\hline$D$ & effective diffusion coefficient $\left[\mathrm{cm}^{2} \mathrm{~s}^{-1}\right]$ \\
\hline$D^{*}$ & $D / \varepsilon\left[\mathrm{cm}^{2} \mathrm{~s}^{-1}\right]$ \\
\hline$D^{\prime}$ & $\begin{array}{l}\text { estimate of effective diffusion coefficient from Monte Carlo } \\
\text { calculations }\left[\mathrm{cm}^{2} \mathrm{~s}^{-1}\right]\end{array}$ \\
\hline$I$ & transmitted X-ray intensity $\left[\mathrm{s}^{-1}\right]$ \\
\hline$I_{0}$ & incident $\mathrm{X}$-ray intensity $\left[\mathrm{s}^{-1}\right]$ \\
\hline$J_{a}$ & flux through $y z$ plane at $x=a\left[\mathrm{~g} \mathrm{~cm}^{-2} \mathrm{~s}^{-1}\right]$ \\
\hline$m_{x}$ & projected mass on $y z$ plane at $x\left[\mathrm{~g} \mathrm{~cm}^{-2}\right]$ \\
\hline$M_{x}$ & mass per unit volume at $x\left[\mathrm{~g} \mathrm{~cm}^{-3}\right]$ \\
\hline$N$ & transmitted X-ray count \\
\hline $\bar{N}$ & mean transmitted X-ray count \\
\hline $\bar{N}_{0}$ & mean transmitted X-ray count in the absence of diffusant \\
\hline $\bar{N}_{0}^{*}$ & mean incident $X$-ray count \\
\hline$t$ & time of diffusion [s] \\
\hline$\Delta t$ & time interval between X-ray absorption measurements [s] \\
\hline$T$ & dimensionless variable, $D^{*} t / A^{2}$ \\
\hline$\delta T, \Delta T$ & dimensionless intervals of $T$ \\
\hline & coordinate axis along the path of diffusion $[\mathrm{cm}]$ \\
\hline$\Delta x$ & distance interval between X-ray absorption measurements $[\mathrm{cm}]$ \\
\hline$X$ & dimensionless variable, $x / A$ \\
\hline$\delta X, \Delta X$ & dimensionless intervals of $X$ \\
\hline$y$ & $\begin{array}{l}\text { coordinate axis normal to diffusio } \\
\text { coordinate axis in direction of X-r }\end{array}$ \\
\hline
\end{tabular}

Greek symbols

fractional volume porosity

mass absorption coefficient $\left[\mathrm{cm}^{2} \mathrm{~g}^{-1}\right]$

density $\left[\mathrm{g} \mathrm{cm}^{-3}\right]$

$\mathrm{X}$-ray counting time $[\mathrm{s}]$

\section{Subscripts}

$\begin{array}{ll}d & \text { diffusing species } \\ f & \text { fluid } \\ F & \text { fluid containing diffusant } \\ \ell & \text { solvent (liquid) } \\ s & \text { solid }\end{array}$

